\title{
A Journey through the Early Evidence Linking Hydration to Metabolic Health
}

\author{
Tiphaine Vanhaecke $^{\mathrm{a}}$ Erica T. Perrier ${ }^{\mathrm{a}}$ Olle Melander ${ }^{\mathrm{b}, \mathrm{c}}$ \\ aHealth, Hydration and Nutrition Science Department, Danone Research, Palaiseau, France; \\ ${ }^{b}$ Department of Clinical Science, Skåne University Hospital, Lund University, Malmö, Sweden; \\ 'Department of Internal Medicine, Skåne University Hospital, Malmö, Sweden
}

\section{Keywords}

Vasopressin · Copeptin · Metabolic health · Diabetes ·

Water $\cdot$ Hydration $\cdot$ Dehydration

\begin{abstract}
The idea that water intake or hydration may play an intrinsic, independent role in modulating metabolic disease risk is relatively recent. Here, we outline the journey from early experimental works to more recent evidence linking water and hydration to metabolic health. It has been known for decades that individuals with existing metabolic dysfunction experience challenges to body water balance and have elevated arginine vasopressin (AVP), a key hormone regulating body fluid homeostasis. Later, intervention studies demonstrated that altering fluid balance in these individuals could worsen their condition, suggesting that hydration played a role in modulating glycemic control. More recently, observational and interventional studies in healthy individuals have implicated the hydration-vasopressin axis in the pathophysiology of metabolic diseases. Individuals with higher AVP (or its surrogate, copeptin) are at higher risk for developing type 2 diabetes and components of the metabolic syndrome, an association that remains even when controlling for known risk factors. Supporting preclinical work also suggests a causal role for AVP in metabolic dysfunction. It is known that individuals who habitually drink less fluids tend to have higher circulating AVP, which may be lowered by increasing water
\end{abstract} www.karger.com/anm

Karger $\frac{1}{\%}$ karger@karger.com
(C) 2021 The Author(s)

Published by S. Karger AG, Basel

This article is licensed under the Creative Commons AttributionNC-ND) (http://www.karger.com/Services/OpenAccessLicense). Usage and distribution for commercial purposes as well as any distribution of modified material requires written permission. NonCommercial-NoDerivatives 4.0 International License (CC BY- intake. In the short term, water supplementation in habitual low drinkers with high copeptin may reduce fasting glucose or glucagon, generating a proof of concept for the role of water supplementation in reducing incident metabolic disease. A large randomized trial is ongoing to determine whether water supplementation for 1 year in subjects with low water intake can meaningfully reduce fasting glucose, risk of new-onset diabetes, and other cardiometabolic risk factors.

(c) 2021 The Author(s)

Published by S. Karger AG, Basel

\section{Introduction}

The pandemic of metabolic disorders and type 2 diabetes, in particular, is one of the major threats to global health of this time. Indeed, metabolic disorders are associated with increased risk of developing serious and lifethreatening complications, require increased need for medical care, and are spreading across all socioeconomic classes and countries [1]. A body of research has demonstrated that lifestyle interventions could help prevent or delay type 2 diabetes in populations at high risk of developing diabetes $[2,3]$. The focus of lifestyle interventions has primarily been on weight reduction through increased physical activity and dietary modifications, for example, reduction of caloric and fat intakes and increased fiber intake. Within this context, replacing sugar- 
and calorie-containing beverages with water is an obvious preventive measure with the potential to reduce incident diabetes $[4,5]$. Drinking plenty of plain water is essential for promoting optimal functioning of the human body and is now considered to be an integral part of a healthy diet for the prevention and management of metabolic disease [1]. However, despite the apparent consensus around its vital role for health, paradoxically the independent role of water as a nutrient in supporting health in the general population has seldom been considered [6]. Recent preliminary evidence suggests that water intake and hydration may have intrinsic effects on glucose homeostasis. Here, we outline the case for water intake, not only as a substitute for calorie-containing beverages but also as a potential independent contributor to metabolic health.

\section{Elevated Arginine Vasopressin, Body Water Disturbance, and Metabolic Dysfunction: Cause or Consequence?}

It is well-known that the hyperglycemia characteristic of diabetes presents unique challenges to body water balance. Elevated blood glucose exerts osmotic pressure which elicits an internal shift in body water from the intracellular to the extracellular space, preserving blood osmolality and volume at the cost of cellular dehydration [7]. Moreover, diabetes can increase risk of dehydration through increased urinary water losses. These disturbances to body water balance are also evident in early empirical observations of the hormone arginine vasopressin (AVP) in individuals with diabetes mellitus. Observations in patients with uncontrolled diabetes show both elevated plasma AVP concentrations and a chronic hypovolemic hyperosmolar state - a consequence of severe glucosuria caused by hyperglycemia [8]. Preclinical studies later confirmed high vasopressin expression and concentrations in animal models of diabetes $[9,10]$. Since the primary role of AVP is to regulate body fluid balance, it is thought that the activation of the fluid regulatory endocrine response in these patients may be an attempt to conserve the volume and osmolality of the vascular compartment. Thus, in existing diabetes, it is far more likely that elevated AVP is a consequence of hyperglycemia and ensuing body water dysregulation, and not the cause of the disease.

However, not only is AVP secreted in excess as a consequence of uncontrolled diabetes, but more recent reports suggest that it may also be a contributing factor to glycemic control. Two studies have reported that alterations to body water balance worsened glucose regulation in diabetic patients $[11,12]$. For instance, in a recent small interventional study in men with type 2 diabetes, 3 days of water restriction resulted in deteriorated glucose response to an oral glucose tolerance test (OGTT), alterations to fasting insulin resistance and sensitivity indices, and reduced whole-body glucose disposal during the OGTT. Furthermore, water restriction resulted in higher plasma cortisol throughout the OGTT [12]. This may suggest that hydration and the hormonal regulation of fluid balance may independently alter glucose regulation in individuals already displaying metabolic dysfunction. While direct evidence in humans is scarce, evidence supporting this hypothesis can be found in preclinical models of metabolic dysfunction. In obese rats, supraphysiologic AVP infusion was shown to promote insulin resistance and to promote glucose intolerance to a glucose challenge [13]. Because AVP is secreted in response to increases in plasma osmolality, replenishing body water by increasing fluid intake may reduce plasma osmolality and, consequently, AVP secretion. Interestingly, increased water intake designed to reduce AVP in this animal model prone to metabolic dysfunction led to an improved glycemic and insulinemic response to an insulin tolerance test, as well as a reduction of liver steatosis, a common complication of obesity. This may suggest that hydration could help reduce some metabolic dysfunction associated with obesity. These data suggest the possibility of a causal involvement of the AVP system in glucose dysregulation, as well as a potential AVP-lowering effect of water associated with decreased metabolic dysfunction.

\section{How Might Arginine Vasopressin and Body Water Regulation Impact Glucose Regulation?}

At least 2 plausible, interrelated physiological systems have been hypothesized to contribute to the mechanisms underlying hydration and glucose regulation. First, AVP secretion may directly modulate glycemic control through actions in the liver and pancreas (Fig. 1). Second, AVP secretion may also act indirectly by stimulating the hypothalamo-pituitary-adrenal (HPA) axis (Fig. 1).

AVP is produced in the hypothalamus and released by the posterior pituitary gland in response to high plasma osmolality, decreased plasma volume, or low blood pressure, with increasing plasma osmolality being the most sensitive stimulus for AVP secretion in the general population $[14,15]$. Vasopressin induces an antidiuretic action 
Fig. 1. The potential mechanisms by which the AVP system may impact glucose metabolism are illustrated. AVP stimulates glucose output by activation of glycogenolysis and gluconeogenesis through activation of the hepatic V1a receptor (V1aR). Vasopressin stimulates the secretion of glucoregulatory hormones (insulin and glucagon) through activation of the pancreatic V1b receptor (V1bR). Vasopressin stimulates ACTH and cortisol secretion through activation of pituitary V1bR and V1aR in the adrenal cortex, respectively, thereby inhibiting insulin-mediated peripheral glucose disposal and prompting hepatic glucose output. AVP, arginine vasopressin; ACTH, adrenocorticotropic hormone.

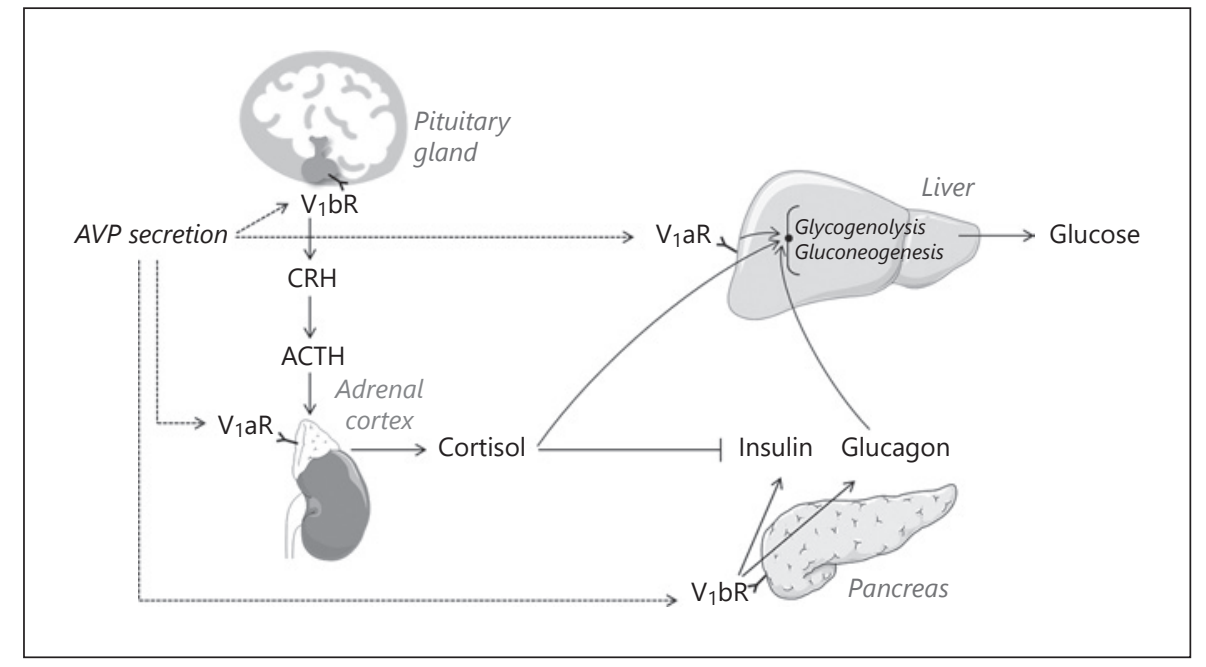

on the kidney through binding to its V2 receptors in the collecting duct. This powerful feedback loop leads to the excretion of smaller volume of a more concentrated urine, thereby countering the increase in plasma osmolality and maintaining body water balance [14]. A series of early experimental and preclinical works have shown that modulating AVP or manipulating the action of its receptors can induce alterations of glycemic and insulinemic control. Activation of the hepatic V1a receptor (V1aR) has been shown to stimulate glycogenolysis and gluconeogenesis, leading to increased plasma glucose concentrations [1618]. In the pancreas, depending upon extracellular levels of glucose, activation of the $\mathrm{V} 1 \mathrm{~b}$ receptor (V1bR) may promote either insulin or glucagon release $[19,20]$. Additionally, coadministration of high AVP and a selective $\mathrm{V} 1 \mathrm{aR}$ antagonist was shown to attenuate glucose intolerance both in obese and normoglycemic rats $[13,21]$. In parallel, AVP acts in synergy with components of the HPA axis, including the corticotropin-releasing hormone to induce adrenocorticotropic hormone secretion from the pituitary gland [22] through binding to the V1bR located in the corticotrophs of the pituitary [23]. Both this activation of the HPA axis and the direct activation of $\mathrm{V} 1 \mathrm{aR}$ in the adrenal cortex [24] are suggested to increase cortisol secretion, eventually prompting gluconeogenesis and inhibiting peripheral glucose disposal [25]. Recent experimental evidence in knockout models of $\mathrm{V} 1 \mathrm{aR}$ and V1bR further confirmed the implication of the vasopressin system in modulating glycemic control. Mice lacking the V1aR display impaired glucose tolerance along with alterations in plasma volume, body weight gain, and energy intake as compared to wild-type mice [26], whereas mice lacking the V1bR display better glycemic control together with enhanced insulin sensitivity [27]. Together, these studies, which manipulated both the hormone and its receptors, provide robust evidence for a direct role of AVP in glucose regulation.

In addition to these 2 mechanisms, it has also been proposed that the activation of the renin-angiotensin-aldosterone system (RAAS) may also contribute to metabolic dysfunction $[16,28]$. This system is activated in response to the reduction of circulating blood volume (hypovolemia) and blood pressure. However, such conditions are unlikely to occur in normal living conditions where it is known that plasma osmolality is the primary stimulus for early compensatory responses to maintain body fluid balance $[14,15]$. This is supported by the recent findings of Johnson et al. [12], who found increased plasma osmolality but no change in RAAS hormones despite alterations to glycemic control in diabetic adults following water restriction. Therefore, an activation of the RAAS appears less plausible than the previous 2 mechanisms in the context of day-to-day regulation of fluid balance and glucose metabolism.

While mechanistic studies are typically more difficult to confirm in humans, recent analytical methods have enabled further insight into a causal role for AVP in glycemic control. Common genetic variation in the human AVP gene or the V1aR gene have been associated with circulating AVP concentration and incidence of hyperglycemia in a French cohort [29] or with slightly higher fasting glucose in a large Swedish cohort and with diabetes prevalence in a subset of subjects of this cohort with high fat intake and elevated BMI [30], respectively. In the 
latter cohort, allelic variance in the human V1bR gene was also associated with higher risk of diabetes development [31], further pointing to an essential role for the vasopressin system in glucose metabolism.

\section{What Evidence Is There for Excessive AVP, Low Fluid Intake, and Metabolic Health in the General Population?}

Previously, we have seen that AVP is elevated in individuals with existing metabolic dysfunction; that alterations to fluid balance may worsen their condition; and that a robust body of preclinical work indicates a causal role for AVP in glycemic control. However, these findings alone do not indicate whether such a relationship exists in healthy individuals or whether AVP and hydration might be a contributing factor in the development of metabolic disease. Recently, several large longitudinal cohorts have examined the association between AVP (measured by its surrogate, copeptin) and the risk of incident diabetes in the general population. High plasma copeptin has been consistently identified as an independent predictor of type 2 diabetes in 4 European cohorts [29, 32-34]. In a Scandinavian cohort, the quartile of the population with the highest plasma concentration of copeptin had a 3.5-fold increased risk of newonset type 2 diabetes compared to the bottom quartile, an association independent of fasting insulin and glucose [32]. Similarly, Roussel and colleagues [29] confirmed the association of plasma copeptin with the incidence of impaired fasting glucose and type 2 diabetes in a French longitudinal cohort with an average 13-year follow-up, after adjusting for baseline water intake fasting insulin and glucose both in men and women, whereas a Dutch cohort reported this independent association in women only [33]. A British cohort of older men with no prevalent diabetes also confirmed that copeptin independently predicted new-onset diabetes in this older population [34]. Less consistent observations have been reported between high plasma copeptin and some metabolic risk factors including abdominal obesity, decreased HDL cholesterol, insulin resistance, and hyperglycemia [34-38].

In addition, 2 proofs of concept studies in healthy humans have reported changes to glycemic control in response to increased AVP. Specifically, supraphysiologic AVP infusion [39] or osmotic stimulation of vasopressin (copeptin) within a high but physiological range [40] has been shown to induce modest but significant increases in glucose and glucagon. In contrast, another study using passive heating to dehydrate participants showed no dif-

Water, Hydration, and Metabolic Health ference in glucose tolerance in the short term [41] despite achieving similar copeptin concentrations to Jansen et al. [40]. Together, these data suggest that elevation of vasopressin (copeptin) concentrations is a risk factor for the development of diabetes in the long term. However, due to the paucity of available data and the variability of trial conditions, it is still unclear whether the activation of the AVP system may alter glucose metabolism in the short term, which conditions would be required, and which mechanisms would be involved.

With vasopressin being the key regulator of body fluid balance, de facto but indirectly connected to fluid intake, other research has investigated the direct links between water intake, glycemia, and incident diabetes. Here, higher water intake has consistently been associated with lower blood glucose levels and risk of diabetes in men, but not in women $[4,42,43]$. While the underlying reasons for possible sex differences in water intake and metabolic dysfunction are not clear, interestingly, AVP (and copeptin) also tends to be higher in men than in women [29, $33,36,44]$. These findings suggest the need for intervention studies to assess potentially differential effects of water intake on metabolic health in men and women.

\section{From Observation to Action: Could Increased Water Intake Be an Actionable Lever to Decrease Metabolic Disease Risk in the General Population?}

In otherwise healthy individuals with chronically higher AVP, might water intake be a simple and effective measure to reduce AVP and thus lower diabetes risk? The rationale for considering water supplementation as a preventive measure comes from observational studies reporting that individuals with low fluid intake have higher AVP and copeptin concentrations as compared to those who consume more fluids despite similar plasma osmolality $[45,46]$. Additionally, there is compelling evidence that increasing water intake can meaningfully reduce AVP (copeptin) over a period of hours, days, or weeks [46-49] and that the copeptin-lowering effect is driven by individuals with the highest baseline copeptin or other signs of insufficient water intake $[47,49]$. Recent shortterm trials suggest this AVP-lowering effect may also impact metabolic endpoints. In a 1-week water intervention study, Enhörning et al. [47] documented a small but significant reduction in plasma glucagon, but not in fasting plasma insulin or glucose, in individuals with the highest baseline copeptin. Separately, a 6-week water intervention in adults with high urine osmolality, low urine vol- 
ume, and high copeptin induced a small but significant decrease in fasting plasma glucose, but no changes in fasting plasma insulin or glucagon [49]. These preliminary findings, despite the inconsistencies in metabolic outcomes, together generate a proof of concept for the role of water supplementation in metabolic disease risk. A large randomized controlled trial to explore whether water supplementation in subjects with low water intake can durably reduce fasting glucose, risk of new-onset diabetes, and other cardiometabolic risk factors is currently ongoing (NCT03422848).

\section{What Is Missing?}

Current evidence points to AVP as an actor in metabolic dysfunction and suggests that water intake may play a role in reducing metabolic disease risk. However, there are a number of areas where research is missing, where results are conflicting, or where the long-term health implications remain unclear. Here, we offer a list of questions that remain partially or wholly unanswered:

- Investigate genetic and physiological characteristics that may influence AVP and its link with disease.

- Distinguish the importance of water and hydration in the primary prevention of metabolic disease, from its importance in the management of existing metabolic dysfunction.

- Understand whether short-term changes in fasting glucose are maintained over time and whether these small but significant changes are meaningful in the long-term management of disease risk.

- Understand which mechanism(s) play a key role on the short and long term, and understand the progres- sion between short-term effects on glucose regulation and long-term metabolic dysregulation.

\section{Conclusion}

A combination of preclinical, observational, and intervention studies point to a direct link between low water intake, increased antidiuretic signal or high AVP (copeptin) concentration, and metabolic dysfunction. While some reports are conflicting, the available evidence would suggest that men may be more at risk, as illustrated by several cohort studies as well as a tendency to have higher circulating AVP. Regardless of sex, individuals with higher circulating AVP and other signs of insufficient hydration appear to be the best candidates for a water intervention to lower AVP and metabolic risk. In these individuals, improving hydration by increasing water intake may provide a simple and inexpensive intervention to help prevent the development of metabolic dysfunction. The promising findings shown in 2 short-term interventions should be further researched with large-scale, longer term studies.

\section{Statement of Ethics}

Ethical conflicts are non-applicable to a review article.

\section{Conflict of Interest Statement}

T.V. and E.P. are full time employee of Danone Research. O.M. received travel expenses and registration fees from Danone Research to attend the 2019 Hydration for Health Scientific Conference, he previously received a research grant and consultancy fees from Danone Research.

\section{References}

1 IDF diabetes atlas. 9th ed. Brussels, Belgium; 2019.

2 Balk EM, Earley A, Raman G, Avendano EA, Pittas AG, Remington PL. Combined diet and physical activity promotion programs to prevent type 2 diabetes among persons at increased risk: a systematic review for the community preventive services task force. Ann Intern Med. 2015;163(6):437-51.

3 Barry E, Roberts S, Oke J, Vijayaraghavan S, Normansell R, Greenhalgh T. Efficacy and effectiveness of screen and treat policies in prevention of type 2 diabetes: systematic review and meta-analysis of screening tests and interventions. BMJ. 2017;356:16538.
4 Pan A, Malik VS, Schulze MB, Manson JE, Willett WC, Hu FB. Plain-water intake and risk of type 2 diabetes in young and middle-aged women. Am J Clin Nutr. 2012;95(6):1454-60.

5 O'Connor L, Imamura F, Lentjes MA, Khaw KT, Wareham NJ, Forouhi NG. Prospective associations and population impact of sweet beverage intake and type 2 diabetes, and effects of substitutions with alternative beverages. Diabetologia. 2015;58(7):1474-83.

6 Rush EC. Water: neglected, unappreciated and under researched. Eur J Clin Nutr. 2013; 67(5):492-5.

7 Singer DL, Drolette ME, Hurwitz D, Freinkel N. Serum osmolality and glucose in maturity onset diabetes mellitus. Arch Intern Med. 1962;110(5):758-62.

8 Zerbe RL, Vinicor F, Robertson GL. Plasma vasopressin in uncontrolled diabetes mellitus. Diabetes. 1979;28(5):503-8.

9 Brooks DP, Nutting DF, Crofton JT, Share L. Vasopressin in rats with genetic and streptozocin-induced diabetes. Diabetes. 1989;38(1): 54-7.

10 Yi SS, Hwang IK, Kim YN, Kim IY, Pak SI, Lee IS, et al. Enhanced expressions of arginine vasopressin (Avp) in the hypothalamic paraventricular and supraoptic nuclei of type 2 diabetic rats. Neurochem Res. 2008;33(5):83341. 
11 Burge MR, Garcia N, Qualls CR, Schade DS. Differential effects of fasting and dehydration in the pathogenesis of diabetic ketoacidosis. Metab Clin Exp. 2001;50(2):171-7.

12 Johnson EC, Bardis CN, Jansen LT, Adams JD, Kirkland TW, Kavouras SA. Reduced water intake deteriorates glucose regulation in patients with type 2 diabetes. Nutr Res. 2017; 43:25-32.

13 Taveau C, Chollet C, Waeckel L, Desposito D, Bichet DG, Arthus MF, et al. Vasopressin and hydration play a major role in the development of glucose intolerance and hepatic steatosis in obese rats. Diabetologia. 2015;58(5): 1081-90.

14 Robertson GL. The regulation of vasopressin function in health and disease. Recent Prog Horm Res. 1976;33:333-85.

15 Kimura T, Minai K, Matsui K, Mouri T, Sato T. Effect of various states of hydration on plasma $\mathrm{ADH}$ and renin in man. J Clin Endocrinol Metab. 1976;42(1):79-87.

16 Hems DA, Rodrigues LM, Whitton PD. Rapid stimulation by vasopressin, oxytocin and angiotensin II of glycogen degradation in hepatocyte suspensions. Biochem J. 1978; 172(2):311-7.

17 Whitton PD, Rodrigues LM, Hems DA. Stimulation by vasopressin, angiotensin and oxytocin of gluconeogenesis in hepatocyte suspensions. Biochem J. 1978;176(3):893-8.

18 Keppens S, de Wulf $\mathrm{H}$. The nature of the hepatic receptors involved in vasopressin-induced glycogenolysis. Biochim Biophys Acta. 1979;588(1):63-9.

19 Lee B, Yang C, Chen TH, al-Azawi N, Hsu WH. Effect of AVP and oxytocin on insulin release: involvement of $\mathrm{V} 1 \mathrm{~b}$ receptors. Am J Physiol. 1995;269(6 Pt 1):E1095-100.

20 Abu-Basha EA, Yibchok-Anun S, Hsu WH. Glucose dependency of arginine vasopressininduced insulin and glucagon release from the perfused rat pancreas. Metab Clin Exp. 2002; 51(9):1184-90.

21 Taveau C, Chollet C, Bichet DG, Velho G, Guillon G, Corbani M, et al. Acute and chronic hyperglycemic effects of vasopressin in normal rats: involvement of V1A receptors. Am J Physiol Endocrinol Metab. 2017;312(3): E127-35.

22 Gillies GE, Linton EA, Lowry PJ. Corticotropin releasing activity of the new CRF is potentiated several times by vasopressin. Nature. 1982;299(5881):355-7.

23 Tanoue A, Ito S, Honda K, Oshikawa S, Kitagawa Y, Koshimizu TA, et al. The vasopressin V1b receptor critically regulates hypothalamic-pituitary-adrenal axis activity under both stress and resting conditions. J Clin Invest. 2004;113(2):302-9.

24 Perraudin V, Delarue C, Lefebvre H, Contesse V, Kuhn JM, Vaudry H. Vasopressin stimulates cortisol secretion from human adrenocortical tissue through activation of $\mathrm{V} 1$ receptors. J Clin Endocrinol Metab. 1993;76(6): $1522-8$
25 Rizza RA, Mandarino LJ, Gerich JE. Cortisolinduced insulin resistance in man: impaired suppression of glucose production and stimulation of glucose utilization due to a postreceptor detect of insulin action. J Clin Endocrinol Metab. 1982;54(1):131-8.

26 Aoyagi T, Birumachi J, Hiroyama M, Fujiwara Y, Sanbe A, Yamauchi J, et al. Alteration of glucose homeostasis in V1a vasopressin receptor-deficient mice. Endocrinology. 2007;148(5):2075-84.

27 Fujiwara Y, Hiroyama M, Sanbe A, Aoyagi T, Birumachi J, Yamauchi J, et al. Insulin hypersensitivity in mice lacking the V1b vasopressin receptor. J Physiol. 2007;584(Pt 1):23544.

28 Joseph JJ, Echouffo Tcheugui JB, Effoe VS, Hsueh WA, Allison MA, Golden SH. Reninangiotensin-aldosterone system, glucose metabolism and incident type 2 diabetes mellitus: MESA. J Am Heart Assoc. 2018;7(17): e009890.

29 Roussel R, El Boustany R, Bouby N, Potier L, Fumeron F, Mohammedi K, et al. Plasma copeptin, AVP gene variants, and incidence of type 2 diabetes in a cohort from the community. J Clin Endocrinol Metab. 2016;101(6): 2432-9.

30 Enhörning S, Leosdottir M, Wallström P, Gullberg B, Berglund G, Wirfält E, et al. Relation between human vasopressin 1a gene variance, fat intake, and diabetes. Am J Clin Nutr. 2009;89(1):400-6.

31 Enhörning S, Sjögren M, Hedblad B, Nilsson PM, Struck J, Melander O. Genetic vasopres$\sin 1 \mathrm{~b}$ receptor variance in overweight and diabetes mellitus. Eur J Endocrinol. 2016; 174(1):69-75.

32 Enhorning S, Wang TJ, Nilsson PM, Almgren P, Hedblad B, Berglund G, et al. Plasma copeptin and the risk of diabetes mellitus. Circulation. 2010;121(19):2102-8.

33 Abbasi A, Corpeleijn E, Meijer E, Postmus D, Gansevoort RT, Gans RO, et al. Sex differences in the association between plasma copeptin and incident type 2 diabetes: the prevention of renal and vascular endstage disease (PREVEND) study. Diabetologia. 2012;55(7): 1963-70.

34 Wannamethee SG, Welsh P, Papacosta O, Lennon L, Whincup PH, Sattar N. Copeptin, insulin resistance, and risk of incident diabetes in older men. J Clin Endocrinol Metab. 2015;100(9):3332-9.

35 Saleem U, Khaleghi M, Morgenthaler NG, Bergmann A, Struck J, Mosley TH Jr, et al. Plasma carboxy-terminal provasopressin (copeptin): a novel marker of insulin resistance and metabolic syndrome. J Clin Endocrinol Metab. 2009;94(7):2558-64.

36 Enhorning S, Struck J, Wirfalt E, Hedblad B, Morgenthaler NG, Melander O. Plasma copeptin, a unifying factor behind the metabolic syndrome. J Clin Endocrinol Metab. 2011; 96(7):E1065-72.
37 Enhörning S, Bankir L, Bouby N, Struck J, Hedblad B, Persson M, et al. Copeptin, a marker of vasopressin, in abdominal obesity, diabetes and microalbuminuria: the prospective Malmö Diet and Cancer Study cardiovascular cohort. Int J Obes. 2013;37(4):598-603.

38 Then C, Kowall B, Lechner A, Meisinger C, Heier M, Koenig W, et al. Plasma copeptin is associated with type 2 diabetes in men but not in women in the population-based KORA F4 study. Acta Diabetol. 2015;52(1):103-12.

39 Spruce BA, McCulloch AJ, Burd J, Orskov H, Heaton A, Baylis PH, et al. The effect of vasopressin infusion on glucose metabolism in man. Clin Endocrinol. 1985;22(4):463-8

40 Jansen LT, Suh H, Adams JD, Sprong CA, Seal $\mathrm{AD}$, Scott DM, et al. Osmotic stimulation of vasopressin acutely impairs glucose regulation: a counterbalanced, crossover trial. Am J Clin Nutr. 2019;110(6):1344-52.

41 Carroll HA, Templeman I, Chen Y-C, Edinburgh RM, Burch EK, Jewitt JT, et al. Effect of acute hypohydration on glycemic regulation in healthy adults: a randomized crossover trial. J Appl Physiol. 2019;126(2):422-30.

42 Roussel R, Fezeu L, Bouby N, Balkau B, Lantieri $\mathrm{O}$, Alhenc-Gelas $\mathrm{F}$, et al. Low water intake and risk for new-onset hyperglycemia. Diabetes Care. 2011;34(12):2551-4

43 Carroll HA, Betts JA, Johnson L. An investigation into the relationship between plain water intake and glycated $\mathrm{Hb}$ (HbAlc): a sexstratified, cross-sectional analysis of the UK National Diet and Nutrition Survey (20082012). Br J Nutr. 2016;116(10):1-11.

44 Morgenthaler NG, Struck J, Alonso C, Bergmann A. Assay for the measurement of copeptin, a stable peptide derived from the precursor of vasopressin. Clin Chem. 2006;52(1): 112-9.

45 Perrier E, Vergne S, Klein A, Poupin M, Rondeau P, Le Bellego L, et al. Hydration biomarkers in free-living adults with different levels of habitual fluid consumption. $\mathrm{Br} \mathrm{J}$ Nutr. 2013;109(9):1678-87.

46 Johnson EC, Muñoz CX, Jimenez L, Le Bellego L, Kupchak BR, Kraemer WJ, et al. Hormonal and thirst modulated maintenance of fluid balance in young women with different levels of habitual fluid consumption. Nutrients. 2016;8(5):302.

47 Enhörning S, Tasevska I, Roussel R, Bouby N, Persson M, Burri P, et al. Effects of hydration on plasma copeptin, glycemia and gluco-regulatory hormones: a water intervention in humans. Eur J Nutr. 2019;58(1):315-24.

48 Lemetais G, Melander O, Vecchio M, Bottin $\mathrm{JH}$, Enhörning S, Perrier ET. Effect of increased water intake on plasma copeptin in healthy adults. Eur J Nutr. 2018;57(5):188390.

49 Enhörning S, Brunkwall L, Tasevska I, Ericson U, Persson M, Lemetais G, et al. Increasing water intake reduces high copeptin in healthy adults. FASEB J. 2018;32(1_Suppl): 597-3. 